\title{
Some new bounds for the minimum eigenvalue of the Hadamard product of an $M$-matrix and an inverse $M$-matrix
}

\section{Yao-tang Li ${ }^{*}$, Feng Wang, Chao-qian Li and Jian-xing Zhao}

\section{"Correspondence:}

liyaotang@ynu.edu.cn

School of Mathematics and

Statistics, Yunnan University,

Kunming, Yunnan 650091, P.R. China

\begin{abstract}
Let $A$ and $B$ be nonsingular $M$-matrices. Several new bounds on the minimum eigenvalue for the Hadamard product of $B$ and the inverse matrix of $A$ are given. These bounds can improve considerably some previous results.

MSC: $15 \mathrm{~A} 42 ; 15 \mathrm{~B} 34$
\end{abstract}

Keywords: $M$-matrix; Hadamard product; minimum eigenvalue

\section{Introduction}

Let $\mathbb{C}^{n \times n}\left(\mathbb{R}^{n \times n}\right)$ denote the set of all $n \times n$ complex (real) matrices, $A=\left(a_{i j}\right) \in \mathbb{C}^{n \times n}, N=$ $\{1,2, \ldots, n\}$. We write $A \geq 0$ if $a_{i j} \geq 0$ for any $i, j \in N$. If $A \geq 0, A$ is called a nonnegative matrix. The spectral radius of $A$ is denoted by $\rho(A)$.

We denote by $Z_{n}$ the class of all $n \times n$ real matrices, whose off-diagonal entries are nonpositive. A matrix $A=\left(a_{i j}\right) \in Z_{n}$ is called a nonsingular $M$-matrix if there exist a nonnegative matrix $B$ and a nonnegative real number $s$ such that $A=s I-B$ with $s>\rho(B)$, where $I$ is the identity matrix. $M_{n}$ will be used to denote the set of all $n \times n$ nonsingular $M$-matrices. Let us denote $\tau(A)=\min \{\operatorname{Re}(\lambda): \lambda \in \sigma(A)\}$, where $\sigma(A)$ denotes the spectrum of $A$.

The Hadamard product of two matrices $A=\left(a_{i j}\right) \in \mathbb{C}^{n \times n}$ and $B=\left(b_{i j}\right) \in \mathbb{C}^{n \times n}$ is the matrix $A \circ B=\left(a_{i j} b_{i j}\right) \in \mathbb{C}^{n \times n}$. If $A, B \in M_{n}$, then $B \circ A^{-1}$ is also an $M$-matrix (see [1]).

Let $A=\left(a_{i j}\right)$ be an $n \times n$ matrix with all diagonal entries being nonzero throughout. For $i, j, k \in N, i \neq j$, denote

$$
\begin{aligned}
& R_{i}=\sum_{j \neq i}\left|a_{i j}\right|, \quad d_{i}=\frac{R_{i}}{\left|a_{i i}\right|} ; \\
& r_{j i}=\frac{\left|a_{j i}\right|}{\left|a_{j j}\right|-\sum_{k \neq j, i}\left|a_{j k}\right|}, \quad r_{i}=\max _{j \neq i}\left\{r_{j i}\right\} ; \\
& m_{j i}=\frac{\left|a_{j i}\right|+\sum_{k \neq j, i}\left|a_{j k}\right| r_{i}}{\left|a_{j j}\right|}, \quad m_{i}=\max _{j \neq i}\left\{m_{i j}\right\} ; \\
& u_{j i}=\frac{\left|a_{j i}\right|+\sum_{k \neq j, i}\left|a_{j k}\right| m_{k i}}{\left|a_{j j}\right|}, \quad u_{i}=\max _{j \neq i}\left\{u_{i j}\right\} .
\end{aligned}
$$

○2013 Li et al.; licensee Springer. This is an Open Access article distributed under the terms of the Creative Commons Attribution License (http://creativecommons.org/licenses/by/2.0), which permits unrestricted use, distribution, and reproduction in any medium, provided the original work is properly cited. 
In 2013, Zhou et al. [2] obtained the following result: If $A=\left(a_{i j}\right) \in M_{n}$ is a strictly row diagonally dominant matrix, $B=\left(b_{i j}\right) \in M_{n}$ and $A^{-1}=\left(\alpha_{i j}\right)$, then

$$
\tau\left(B \circ A^{-1}\right) \geq \min _{i \in N}\left\{\frac{b_{i i}-m_{i} \sum_{j \neq i}\left|b_{j i}\right|}{a_{i i}}\right\}
$$

In 2013, Cheng et al. [3] presented the following result: If $A=\left(a_{i j}\right) \in M_{n}$ and $A^{-1}=\left(\alpha_{i j}\right)$ is a doubly stochastic matrix, then

$$
\tau\left(A \circ A^{-1}\right) \geq \min _{1 \leq i \leq n}\left\{\frac{a_{i i}-u_{i} \sum_{j \neq i}\left|a_{j i}\right|}{1+\sum_{j \neq i} u_{j i}}\right\} .
$$

In this paper, we present some new lower bounds of $\tau\left(B \circ A^{-1}\right)$ and $\tau\left(A \circ A^{-1}\right)$, which improve (1) and (2).

\section{Main results}

In this section, we present our main results. Firstly, we give some lemmas.

Lemma 1 [4] Let $A=\left(a_{i j}\right) \in \mathbb{R}^{n \times n}$. If $A$ is a strictly row diagonally dominant matrix, then $A^{-1}=\left(\alpha_{i j}\right)$ satisfies

$$
\left|\alpha_{j i}\right| \leq d_{j}\left|\alpha_{i i}\right|, \quad j, i \in N, j \neq i
$$

Lemma 2 Let $A=\left(a_{i j}\right) \in \mathbb{R}^{n \times n}$. If $A$ is a strictly row diagonally dominant $M$-matrix, then $A^{-1}=\left(\alpha_{i j}\right)$ satisfies

$$
\alpha_{j i} \leq w_{j i} \alpha_{i i}, \quad j, i \in N, j \neq i,
$$

where

$$
w_{j i}=\frac{\left|a_{j i}\right|+\sum_{k \neq j, i}\left|a_{j k}\right| m_{k i} h_{i}}{\left|a_{j j}\right|}, \quad h_{i}=\max _{j \neq i}\left\{\frac{\left|a_{j i}\right|}{\left|a_{j j}\right| m_{j i}-\sum_{k \neq j, i}\left|a_{j k}\right| m_{k i}}\right\} .
$$

Proof This proof is similar to the one of Lemma 2.2 in [3].

Lemma 3 If $A=\left(a_{i j}\right) \in M_{n}$ and $A^{-1}=\left(\alpha_{i j}\right)$ is a doubly stochastic matrix, then

$$
\alpha_{i i} \geq \frac{1}{1+\sum_{j \neq i} w_{j i}}, \quad i \in N \text {, }
$$

where $w_{j i}$ is defined as in Lemma 2.

Proof This proof is similar to the one of Lemma 3.1 in [3].

Lemma 4 [4] If $A=\left(a_{i j}\right) \in \mathbb{R}^{n \times n}$ is a strictly row diagonally dominant $M$-matrix, then, for $A^{-1}=\left(\alpha_{i j}\right)$,

$$
\alpha_{i i} \geq \frac{1}{a_{i i}}, \quad i \in N
$$


Lemma 5 [5] If $A=\left(a_{i j}\right) \in \mathbb{C}^{n \times n}$ and $x_{1}, x_{2}, \ldots, x_{n}$ are positive real numbers, then all the eigenvalues of $A$ lie in the region

$$
\bigcup_{i \neq j}\left\{z \in \mathbb{C}:\left|z-a_{i i}\right| \leq x_{i} \sum_{k \neq i} \frac{1}{x_{k}}\left|a_{k i}\right|, i \in N\right\} .
$$

Lemma 6 [6] If $A=\left(a_{i j}\right) \in \mathbb{C}^{n \times n}$ and $x_{1}, x_{2}, \ldots, x_{n}$ are positive real numbers, then all the eigenvalues of $A$ lie in the region

$$
\bigcup_{i \neq j}\left\{z \in \mathbb{C}:\left|z-a_{i i}\right|\left|z-a_{j j}\right| \leq\left(x_{i} \sum_{k \neq i} \frac{1}{x_{k}}\left|a_{k i}\right|\right)\left(x_{j} \sum_{k \neq j} \frac{1}{x_{k}}\left|a_{k j}\right|\right), i, j \in N\right\} .
$$

Theorem 1 If $A=\left(a_{i j}\right), B=\left(b_{i j}\right) \in M_{n}$ and $A^{-1}=\left(\alpha_{i j}\right)$, then

$$
\tau\left(B \circ A^{-1}\right) \geq \min _{1 \leq i \leq n}\left\{\frac{b_{i i}-w_{i} \sum_{j \neq i}\left|b_{j i}\right|}{a_{i i}}\right\}
$$

where $w_{i}=\max _{j \neq i}\left\{w_{i j}\right\}$ and $w_{i j}$ is defined as in Lemma 2 .

Proof It is evident that the result holds with equality for $n=1$.

We next assume that $n \geq 2$.

Since $A$ is an $M$-matrix, there exists a positive diagonal matrix $D$ such that $D^{-1} A D$ is a strictly row diagonally dominant $M$-matrix, and

$$
\tau\left(B \circ A^{-1}\right)=\tau\left(D^{-1}\left(B \circ A^{-1}\right) D\right)=\tau\left(B \circ\left(D^{-1} A D\right)^{-1}\right) .
$$

Therefore, for convenience and without loss of generality, we assume that $A$ is a strictly row diagonally dominant matrix.

(i) First, we assume that $A$ and $B$ are irreducible matrices. Then, for any $i \in N$, we have $0<w_{i}<1$. Since $\tau\left(B \circ A^{-1}\right)$ is an eigenvalue of $B \circ A^{-1}$, then by Lemma 2 and Lemma 5 , there exists an $i$ such that

$$
\begin{aligned}
\left|\tau\left(B \circ A^{-1}\right)-b_{i i} \alpha_{i i}\right| & \leq w_{i} \sum_{j \neq i} \frac{1}{w_{j}}\left|b_{j i} \alpha_{j i}\right| \leq w_{i} \sum_{j \neq i} \frac{1}{w_{j}}\left|b_{j i}\right| w_{j i}\left|\alpha_{i i}\right| \\
& \leq w_{i} \sum_{j \neq i} \frac{1}{w_{j}}\left|b_{j i}\right| w_{j}\left|\alpha_{i i}\right|=w_{i}\left|\alpha_{i i}\right| \sum_{j \neq i}\left|b_{j i}\right| .
\end{aligned}
$$

By Lemma 4 , the above inequality and $0 \leq \tau\left(B \circ A^{-1}\right) \leq b_{i i} \alpha_{i i}$, for any $i \in N$, we obtain

$$
\left|\tau\left(B \circ A^{-1}\right)\right| \geq b_{i i} \alpha_{i i}-w_{i}\left|\alpha_{i i}\right| \sum_{j \neq i}\left|b_{j i}\right| \geq \frac{b_{i i}-w_{i} \sum_{j \neq i}\left|b_{j i}\right|}{a_{i i}} \geq \min _{1 \leq i \leq n}\left\{\frac{b_{i i}-w_{i} \sum_{j \neq i}\left|b_{j i}\right|}{a_{i i}}\right\} .
$$

(ii) Now, assume that one of $A$ and $B$ is reducible. It is well known that a matrix in $Z_{n}$ is a nonsingular $M$-matrix if and only if all its leading principal minors are positive (see [7]). If we denote by $T=\left(t_{i j}\right)$ the $n \times n$ permutation matrix with $t_{12}=t_{23}=\cdots=t_{n-1, n}=t_{n 1}=1$, the remaining $t_{i j}$ zero, then both $A-\epsilon T$ and $B-\epsilon T$ are irreducible nonsingular $M$-matrices for any chosen positive real number $\epsilon$ sufficiently small such that all the leading principal 
minors of both $A-\epsilon T$ and $B-\epsilon T$ are positive. Now, we substitute $A-\epsilon T$ and $B-\epsilon T$ for $A$ and $B$, respectively, in the previous case, and then letting $\epsilon \rightarrow 0$, the result follows by continuity.

From Lemma 3 and Theorem 1, we can easily obtain the following corollaries.

Corollary 1 If $A=\left(a_{i j}\right), B=\left(b_{i j}\right) \in M_{n}$ and $A^{-1}=\left(\alpha_{i j}\right)$ is a doubly stochastic matrix, then

$$
\tau\left(B \circ A^{-1}\right) \geq \min _{1 \leq i \leq n}\left\{\frac{b_{i i}-w_{i} \sum_{j \neq i}\left|b_{j i}\right|}{1+\sum_{j \neq i} w_{j i}}\right\} .
$$

Corollary 2 If $A=\left(a_{i j}\right) \in M_{n}$ and $A^{-1}=\left(\alpha_{i j}\right)$ is a doubly stochastic matrix, then

$$
\tau\left(A \circ A^{-1}\right) \geq \min _{1 \leq i \leq n}\left\{\frac{a_{i i}-w_{i} \sum_{j \neq i}\left|a_{j i}\right|}{1+\sum_{j \neq i} w_{j i}}\right\} .
$$

Remark 1 We next give a simple comparison between (3) and (1), (4) and (2), respectively. Since $m_{j i} h_{i} \leq r_{i}, 0 \leq h_{i} \leq 1, j, i \in N, j \neq i$, then $w_{j i} \leq m_{j i}, w_{i} \leq m_{i}$ and $w_{j i} \leq u_{j i}, w_{i} \leq u_{i}$ for any $j, i \in N, j \neq i$. Therefore,

$$
\begin{aligned}
& \tau\left(B \circ A^{-1}\right) \geq \min _{1 \leq i \leq n}\left\{\frac{b_{i i}-w_{i} \sum_{j \neq i}\left|b_{j i}\right|}{a_{i i}}\right\} \geq \min _{1 \leq i \leq n}\left\{\frac{b_{i i}-m_{i} \sum_{j \neq i}\left|b_{j i}\right|}{a_{i i}}\right\}, \\
& \tau\left(A \circ A^{-1}\right) \geq \min _{1 \leq i \leq n}\left\{\frac{a_{i i}-w_{i} \sum_{j \neq i}\left|a_{j i}\right|}{1+\sum_{j \neq i} w_{j i}}\right\} \geq \min _{1 \leq i \leq n}\left\{\frac{a_{i i}-u_{i} \sum_{j \neq i}\left|a_{j i}\right|}{1+\sum_{j \neq i} u_{j i}}\right\} .
\end{aligned}
$$

So, the bound in (3) is bigger than the bound in (1) and the bound in (4) is bigger than the bound in (2).

Theorem 2 If $A=\left(a_{i j}\right), B=\left(b_{i j}\right) \in M_{n}$ and $A^{-1}=\left(\alpha_{i j}\right)$, then

$$
\begin{aligned}
\tau\left(B \circ A^{-1}\right) \geq & \min _{i \neq j} \frac{1}{2}\left\{\alpha_{i i} b_{i i}+\alpha_{j j} b_{i j}-\left[\left(\alpha_{i i} b_{i i}-\alpha_{j j} b_{j j}\right)^{2}\right.\right. \\
& \left.\left.+4\left(w_{i} \sum_{k \neq i}\left|b_{k i}\right| \alpha_{i i}\right)\left(w_{j} \sum_{k \neq j}\left|b_{k j}\right| \alpha_{j j}\right)\right]^{\frac{1}{2}}\right\},
\end{aligned}
$$

where $w_{i}(i \in N)$ is defined as in Theorem 1.

Proof It is evident that the result holds with equality for $n=1$.

We next assume that $n \geq 2$. For convenience and without loss of generality, we assume that $A$ is a strictly row diagonally dominant matrix.

(i) First, we assume that $A$ and $B$ are irreducible matrices. Let $R_{j}^{\sigma}=\sum_{k \neq j}\left|a_{j k}\right| m_{k i} h_{i}, j, i \in$ $N, j \neq i$. Then, for any $j, i \in N, j \neq i$, we have

$$
R_{j}^{\sigma}=\sum_{k \neq j}\left|a_{j k}\right| m_{k i} h_{i} \leq\left|a_{j i}\right|+\sum_{k \neq j, i}\left|a_{j k}\right| m_{k i} h_{i} \leq R_{j}<a_{j j} .
$$


Therefore, there exists a real number $z_{j i}\left(0 \leq z_{j i} \leq 1\right)$ such that

$$
\left|a_{j i}\right|+\sum_{k \neq j, i}\left|a_{j k}\right| m_{k i} h_{i}=z_{j i} R_{j}+\left(1-z_{j i}\right) R_{j}^{\sigma}, \quad j, i \in N, j \neq i .
$$

Hence,

$$
w_{j i}=\frac{z_{j i} R_{j}+\left(1-z_{j i}\right) R_{j}^{\sigma}}{a_{j j}}, \quad j \in N
$$

Let $z_{j}=\max _{i \neq j} z_{j i}$. Obviously, $0<z_{j} \leq 1$ (if $z_{j}=0$, then $A$ is reducible, which is a contradiction). Let

$$
w_{j}=\max _{i \neq j}\left\{w_{j i}\right\}=\frac{z_{j} R_{j}+\left(1-z_{j}\right) R_{j}^{\sigma}}{a_{j j}}, \quad j \in N .
$$

Since $A$ is irreducible, then $R_{j}>0, R_{j}^{\sigma} \geq 0$, and $0<w_{j}<1$. Let $\tau\left(B \circ A^{-1}\right)=\lambda$. By Lemma 6, there exist $i_{0}, j_{0} \in N, i_{0} \neq j_{0}$ such that

$$
\left|\lambda-\alpha_{i_{0} i_{0}} b_{i_{0} i_{0}}\right|\left|\lambda-\alpha_{j_{0} j_{0}} b_{j_{0} j_{0}}\right| \leq\left(w_{i_{0}} \sum_{k \neq i_{0}} \frac{1}{w_{k}}\left|\alpha_{k i_{0}} b_{k i_{0}}\right|\right)\left(w_{j_{0}} \sum_{k \neq j_{0}} \frac{1}{w_{k}}\left|\alpha_{k j_{0}} b_{k j_{0}}\right|\right) .
$$

And by Lemma 2, we have

$$
\begin{array}{r}
\left(w_{i_{0}} \sum_{k \neq i_{0}} \frac{1}{w_{k}}\left|\alpha_{k i_{0}} b_{k i_{0}}\right|\right)\left(w_{j_{0}} \sum_{k \neq j_{0}} \frac{1}{w_{k}}\left|\alpha_{k j_{0}} b_{k j_{0}}\right|\right) \\
\leq\left(w_{i_{0}} \sum_{k \neq i_{0}}\left|b_{k i_{0}}\right| \alpha_{i_{0} i_{0}}\right)\left(w_{j_{0}} \sum_{k \neq j_{0}}\left|b_{k j_{0}}\right| \alpha_{j_{0} j_{0}}\right) .
\end{array}
$$

Therefore,

$$
\left|\lambda-\alpha_{i_{0} i_{0}} b_{i_{0} i_{0}}\right|\left|\lambda-\alpha_{j_{0} j_{0}} b_{j_{0} j_{0}}\right| \leq\left(w_{i_{0}} \sum_{k \neq i_{0}}\left|b_{k i_{0}}\right| \alpha_{i_{0} i_{0}}\right)\left(w_{j_{0}} \sum_{k \neq j_{0}}\left|b_{k j_{0}}\right| \alpha_{j_{0} j_{0}}\right) .
$$

Furthermore, we obtain

$$
\begin{aligned}
\lambda \geq & \frac{1}{2}\left\{\alpha_{i_{0} i_{0}} b_{i_{0} i_{0}}+\alpha_{j_{0} j_{0}} b_{j_{0} j_{0}}-\left[\left(\alpha_{i_{0} i_{0}} b_{i_{0} i_{0}}-\alpha_{j_{0} j_{0}} b_{j_{0} j_{0}}\right)^{2}\right.\right. \\
& \left.\left.+4\left(w_{i_{0}} \sum_{k \neq i_{0}}\left|b_{k i_{0}}\right| \alpha_{i_{0} i_{0}}\right)\left(w_{j_{0}} \sum_{k \neq j_{0}}\left|b_{k j_{0}}\right| \alpha_{j_{0} j_{0}}\right)\right]^{\frac{1}{2}}\right\},
\end{aligned}
$$

that is,

$$
\begin{aligned}
& \tau\left(B \circ A^{-1}\right) \\
& \quad \geq \frac{1}{2}\left\{\alpha_{i_{0} i_{0}} b_{i_{0} i_{0}}+\alpha_{j_{0} j_{0}} b_{j_{0} j_{0}}-\left[\left(\alpha_{i_{0} i_{0}} b_{i_{0} i_{0}}-\alpha_{j_{0} j_{0}} b_{j_{0} j_{0}}\right)^{2}\right.\right.
\end{aligned}
$$




$$
\begin{aligned}
& \left.\left.+4\left(w_{i_{0}} \sum_{k \neq i_{0}}\left|b_{k i_{0}}\right| \alpha_{i_{0} i_{0}}\right)\left(w_{j_{0}} \sum_{k \neq j 0}\left|b_{k j_{0}}\right| \alpha_{j_{0} j_{0}}\right)\right]^{\frac{1}{2}}\right\} \\
\geq & \min _{i \neq j} \frac{1}{2}\left\{\alpha_{i i} b_{i i}+\alpha_{j j} b_{j j}-\left[\left(\alpha_{i i} b_{i i}-\alpha_{j j} b_{j j}\right)^{2}+4\left(w_{i} \sum_{k \neq i}\left|b_{k i}\right| \alpha_{i i}\right)\left(w_{j} \sum_{k \neq j}\left|b_{k j}\right| \alpha_{j j}\right)\right]^{\frac{1}{2}}\right\} .
\end{aligned}
$$

(ii) Now, assume that one of $A$ and $B$ is reducible. We substitute $A-\epsilon T$ and $B-\epsilon T$ for $A$ and $B$, respectively, in the previous case, and then letting $\epsilon \rightarrow 0$, the result follows by continuity.

Corollary 3 If $A=\left(a_{i j}\right) \in M_{n}$ and $A^{-1}=\left(\alpha_{i j}\right)$, then

$$
\begin{aligned}
\tau\left(A \circ A^{-1}\right) \geq & \min _{i \neq j} \frac{1}{2}\left\{\alpha_{i i} a_{i i}+\alpha_{j j} a_{j j}-\left[\left(\alpha_{i i} a_{i i}-\alpha_{j j} a_{j j}\right)^{2}\right.\right. \\
& \left.\left.+4\left(w_{i} \sum_{k \neq i}\left|a_{k i}\right| \alpha_{i i}\right)\left(w_{j} \sum_{k \neq j}\left|a_{k j}\right| \alpha_{j j}\right)\right]^{\frac{1}{2}}\right\} .
\end{aligned}
$$

Example 1 Let

$$
A=\left(\begin{array}{cccccccccc}
39 & -16 & -2 & -3 & -2 & -5 & -2 & -3 & -5 & 0 \\
-26 & 44 & -2 & -4 & -2 & -1 & 0 & -2 & -3 & -3 \\
-1 & -9 & 29 & -3 & -4 & 0 & -5 & -4 & -1 & -1 \\
-2 & -3 & -10 & 36 & -12 & 0 & -5 & -1 & -2 & 0 \\
0 & -3 & -1 & -9 & 44 & -16 & -3 & -4 & -4 & -3 \\
-3 & -4 & -3 & -4 & -12 & 48 & -18 & -1 & 0 & -2 \\
-2 & -1 & -4 & -3 & -4 & -16 & 45 & -9 & -4 & -1 \\
-1 & -2 & -2 & -2 & -3 & -1 & -5 & 38 & -20 & -1 \\
-2 & -1 & 0 & -3 & -4 & -5 & -2 & -10 & 47 & -19 \\
-1 & -4 & -4 & -4 & 0 & -3 & -4 & -3 & -7 & 31
\end{array}\right),
$$

It is easily proved that $A$ and $B$ are nonsingular $M$-matrices and $A$ is a doubly stochastic matrix.

(i) If we apply Theorem 4.8 of [2], we have

$$
\tau\left(B \circ A^{-1}\right) \geq \min _{1 \leq i \leq n}\left\{\frac{b_{i i}-m_{i} \sum_{j \neq i}\left|b_{j i}\right|}{a_{i i}}\right\}=0.0027
$$


If we apply Theorem 2.4 of [8], we have

$$
\tau\left(B \circ A^{-1}\right) \geq\left(1-\rho\left(J_{A}\right) \rho\left(J_{B}\right)\right) \min _{1 \leq i \leq n} \frac{a_{i i}}{b_{i i}}=0.3485 .
$$

But, if we apply Theorem 1, we have

$$
\tau\left(B \circ A^{-1}\right) \geq \min _{1 \leq i \leq n}\left\{\frac{b_{i i}-w_{i} \sum_{j \neq i}\left|b_{j i}\right|}{a_{i i}}\right\}=0.0435
$$

If we apply Corollary 1, we have

$$
\tau\left(B \circ A^{-1}\right) \geq \min _{1 \leq i \leq n}\left\{\frac{b_{i i}-w_{i} \sum_{j \neq i}\left|b_{j i}\right|}{1+\sum_{j \neq i} w_{j i}}\right\}=0.2172 .
$$

If we apply Theorem 2, we have

$$
\begin{aligned}
\tau\left(B \circ A^{-1}\right) \geq & \min _{i \neq j} \frac{1}{2}\left\{\alpha_{i i} b_{i i}+\alpha_{j j} b_{j j}-\left[\left(\alpha_{i i} b_{i i}-\alpha_{j j} b_{j j}\right)^{2}\right.\right. \\
& \left.\left.+4\left(w_{i} \sum_{k \neq i}\left|b_{k i}\right| \alpha_{i i}\right)\left(w_{j} \sum_{k \neq j}\left|b_{k j}\right| \alpha_{j j}\right)\right]^{\frac{1}{2}}\right\} \\
= & 0.7212 .
\end{aligned}
$$

(ii) If we apply Theorem 3.2 of [3], we get

$$
\tau\left(A \circ A^{-1}\right) \geq \min _{1 \leq i \leq n}\left\{\frac{a_{i i}-u_{i} \sum_{j \neq i}\left|a_{j i}\right|}{1+\sum_{j \neq i} u_{j i}}\right\}=0.3269
$$

But, if we apply Corollary 2, we get

$$
\tau\left(A \circ A^{-1}\right) \geq \min _{1 \leq i \leq n}\left\{\frac{a_{i i}-w_{i} \sum_{j \neq i}\left|a_{j i}\right|}{1+\sum_{j \neq i} w_{j i}}\right\}=0.3605
$$

If we apply Corollary 3, we get

$$
\begin{aligned}
\tau\left(A \circ A^{-1}\right) \geq & \min _{i \neq j} \frac{1}{2}\left\{\alpha_{i i} a_{i i}+\alpha_{j j} a_{j j}-\left[\left(\alpha_{i i} a_{i i}-\alpha_{j j} a_{j j}\right)^{2}\right.\right. \\
& \left.\left.+4\left(w_{i} \sum_{k \neq i}\left|b_{k i}\right| \alpha_{i i}\right)\left(w_{j} \sum_{k \neq j}\left|b_{k j}\right| \alpha_{j j}\right)\right]^{\frac{1}{2}}\right\} \\
= & 0.4072 .
\end{aligned}
$$




\section{Acknowledgements}

The authors are very indebted to the referees for their valuable comments and corrections, which improved the original manuscript of this paper. This work was supported by the National Natural Science Foundation of China (11361074), IRTSTYN and Foundation of Yunnan University (2012CG017).

Received: 27 July 2013 Accepted: 27 September 2013 Published: 07 Nov 2013

\section{References}

1. Fiedler, M, Markham, T: An inequality for the Hadamard product of an M-matrix and inverse M-matrix. Linear Algebra Appl. 101, 1-8 (1988)

2. Zhou, DM, Chen, GL, Wu, GX, Zhang, XY: On some new bounds for eigenvalues of the Hadamard product and the Fan product of matrices. Linear Algebra Appl. 438, 1415-1426 (2013)

3. Cheng, GH, Tan, Q, Wang, ZD: Some inequalities for the minimum eigenvalue of the Hadamard product of an M-matrix and its inverse. J. Inequal. Appl. 2013(65), 1-9 (2013)

4. Yong, XR, Wang, Z: On a conjecture of Fiedler and Markham. Linear Algebra Appl. 288, 259-267 (1999)

5. Varga, RS: Minimal Gerschgorin sets. Pac. J. Math. 15, 719-729 (1965)

6. Horn, RA, Johnson, CR: Matrix Analysis. Cambridge University Press, Cambridge (1985)

7. Berman, A, Plemmons, RJ: Nonnegative Matrices in the Mathematical Sciences. SIAM, Philadelphia (1994)

8. Zhou, DM, Chen, GL, Wu, GX, Zhang, XY: Some inequalities for the Hadamard product of an M-matrix and an inverse M-matrix. J. Inequal. Appl. 2013(16), 1-10 (2013)

10.1186/1029-242X-2013-480

Cite this article as: Li et al.: Some new bounds for the minimum eigenvalue of the Hadamard product of an M-matrix and an inverse M-matrix. Journal of Inequalities and Applications 2013, 2013:480

\section{Submit your manuscript to a SpringerOpen ${ }^{\mathcal{O}}$ journal and benefit from:}

- Convenient online submission

- Rigorous peer review

- Immediate publication on acceptance

- Open access: articles freely available online

- High visibility within the field

- Retaining the copyright to your article 